\title{
Decomposing Socioeconomic Inequality Determinants in Suicide Deaths in Iran: A Concentration Index Approach
}

\author{
Yousef Veisani', Ali Delpisheh" ${ }^{2, *}$, Kourosh Sayehmiri ${ }^{3}$, Ghobad Moradi ${ }^{4}$, Jafar Hassanzadeh ${ }^{5}$ \\ 'Psychosocial Injuries Research Center, Ilam University of Medical Sciences, Ilam, Iran \\ ${ }^{2}$ Department of Clinical Epidemiology, Ilam University of Medical Sciences, Ilam, Iran \\ ${ }^{3}$ Biostatistics, Social Medicine Department, Ilam University of Medical Sciences, Ilam, Iran \\ ${ }^{4}$ Social Determinants of Health Research Center, Kurdistan University of Medical Sciences, Sanandaj, Iran \\ ${ }^{5}$ Research Center for Health Sciences, Department of Epidemiology, School of Health, Shiraz University of Medical Sciences, Shiraz, Iran
}

Background: It is recognized that socioeconomic status (SES) has a significant impact on health and wellbeing; however, the effect of SES on suicide is contested. This study explored the effect of SES in suicide deaths and decomposed inequality into its determinants to calculate relative contributions.

Methods: Through a cross-sectional study, 546 suicide deaths and 6,818 suicide attempts from January 1, 2010 to December 31, 2014 in Ilam Province, Western Iran were explored. Inequality was measured by the absolute concentration index (ACI) and decomposed contributions were identified. All analyses were performed using STATA ver. 11.2 (Stata Corp., College Station, TX, USA).

Results: The overall ACI for suicide deaths was -0.352 (95\% confidence interval, -0.389 to -0.301 ). According to the results, $9.8 \%$ of socioeconomic inequality in suicide deaths was due to addiction in attempters. ACI ranged from -0.34 to -0.03 in 2010-2014, showing that inequality in suicide deaths declined over time.

Conclusion: Findings showed suicide deaths were distributed among the study population unequally, and our results confirmed a gap between advantaged and disadvantaged attempters in terms of death. Socioeconomic inequalities in suicide deaths tended to diminish over time, as suicide attempts progressed in Ilam Province.

Keywords: Inequality; Concentration Index; Socioeconomic Factors; Attempted Suicide 


\section{INTRODUCTION}

Suicide (an act of intentionally terminating one's own life) remains an important public health problem in Western countries and the second leading cause of death for individuals between the ages of 15 and 19 years. ${ }^{1)}$ A systematic review in 2014 by the World Health Organization reported that the incidence of suicide ranged from 0.55 to 5.4 per 100,000 people in the Eastern Mediterranean Region. ${ }^{2)}$

Predictors and risk factors of suicide attempts (a self-initiated act with the presence of intent to die) include prior suicide attempts (history of previous suicide attempts), and certain demographic variables such as unemployment, clinical symptoms, and issues related to medical and social support. ${ }^{3)}$ Risk of death after prior suicide attempt is 3040 times compared with the general population. ${ }^{4)}$ Therefore, a vital step in reducing suicide deaths is the development of a screening program to identify persons at high risk. The coexistence of prior suicide attempts with other risk factors such as psychiatric disorders (specifically major depression) and substance misuse can be related to an increased risk of death from suicide. ${ }^{5)}$ Thus, reducing the trend of higher suicide rates among these most vulnerable populations is an area in need of further study. ${ }^{6}$ )

It is recognized that socioeconomic status (SES) has a significant impact on health and wellbeing. ${ }^{7,8)}$ The relationship between SES and risk of suicide death has been shown in previous reports. ${ }^{9}$ However, findings regarding the effect of SES on suicide have been inconsistent. Some have found that a lower SES increases the risk of suicide death. ${ }^{10,11)}$ While other results oppose this finding. ${ }^{12)}$ These conflicting results could be due to varying methods being used to measure SES.

The mechanism by which lower SES impacts the risk of suicide death is important to understand, to better inform public health policy and plan more effective suicide prevention programs. It may be that individuals with a lower SES experience higher levels of adversity/ stress and fewer opportunities in life, which could increase their susceptibility to suicide attempts and, therefore, increase their risk of suicide death. ${ }^{13)}$

Calculating socioeconomic inequality in health is a common practice worldwide (particularly in developed countries). Reduction of socioeconomic inequality is an achievable goal for coming decades; however, research must provide information necessary to understand this inequality. The concentration index is one common measure to assess health inequality, which can be decomposed into its determinants to calculate their specific contributions.

Therefore, this study was conducted to identify socioeconomic inequality in suicide deaths in Ilam Province, Iran, and to decompose this inequality into its determinants to identify their proportional contributions to the inequality.

\section{METHODS}

Through a cross-sectional study, data on identified suicides were extracted from systematic registration suicide data (SRSD), ${ }^{14)}$ a system supported by Ilam University of Medical Sciences since 2010. Through SRSD, suicide data have been collected daily from medical centers and compared monthly to data from the Iranian Legal Medicine Organization. Overall, data on 546 suicide deaths and 6,818 attempted suicides from March 21, 2010 to December 11, 2014 were included in this study. In the current study, suicide attempts were defined as cases with nonfatal suicide attempt, and suicide deaths were defined as cases in which persons died from suicide. Data for the present study consisted of all suicide attempts in Ilam Province, Iran by residents aged six years or older, who were admitted to the healthcare center during the period of the study. In SRSD, suicide attempts were determined through an analysis of physician claims and hospital admission records. In addition, daily suicide counts were recorded with respect to nine variables: age, sex, marital status, educational level, employment status, partner's employment status, partner's educational level, region of residence, and race. Data concerning mental disorders, addiction, methods of suicide, and outcome were collected from individual outpatient visits on a monthly basis.

\section{Definitions of Variables}

The outcome variable was suicide deaths and independent variables included age at the time of suicide (6-15, 16-25, 26-35, 36-45, 46-55, 56-65, >65 years old), sex, educational level (illiterate, primary school, high school, diploma, university), residence (urban/rural), employment status (homemaker, unemployed, student, employed and other occupation), prior suicide attempt, addiction (alcohol addiction, substance abuse), and mental disorders (depression, anxiety disorders, schizophrenia, eating disorders), and SES. We calculated the SES index by applying principal component analysis (PCA) to the eight SES constituent items: sex, educational level, partner's educational level, marital status, employment status, partner's job, location of residence, and financial status.

\section{Statistical Analysis}

In the present study, SES was calculated using the PCA procedure to identify variables with a greater impact on the whole variance. Using this procedure, new variables that represented SES were identified. ${ }^{15)}$ We used the absolute concentration index (ACI) to measure inequality in suicide death. According to Wagstaff et al., ${ }^{16)}$ the ACI is defined as twice the area between the concentration curve and the diagonal, ranging from - 1 to +1 . The value of ACI measures the severity of socioeconomic inequality: the larger the absolute value of ACI, the greater the disparity. ACI equals zero when the concentration curve coincides with the diagonal, indicating that there is no socioeconomic inequality in suicide deaths. If the curve lies above the diagonal $(\mathrm{ACI}<0)$, this suggests that suicide deaths are more concentrated among lower SES groups. If the concentration curve lies below the diagonal line ( $\mathrm{ACI}>0$ ), this indicates that suicide deaths are more concentrated in higher SES groups. Thus, ACIs provide a clear visual depiction of socioeconomic inequality of suicide deaths. ${ }^{17)}$

The decomposition approach proposed by Wagstaff et al. ${ }^{18)}$ allows 
one to determine the rate of participation of variables in inequality. Thus, we used the decomposition approach to decompose socioeconomic inequality in suicide deaths. In the first step, binary variables were created to determine the rate of participation in inequality. A linear model was used to obtain a predicted model of the outcome variable. In the next step, we identified the contribution of each determinant to the inequality by multiplying the elasticity of each determinant by its concentration index. This was the absolute contribution of each determinant. In the final step, we calculated the percentage contribution of each determinant by dividing its absolute contribution by the concentration index of suicide deaths. ${ }^{19)}$ Multiple logistic regression analysis was used to measure the association between sociodemographic characteristics and suicide behaviors. All analyses were performed at 0.05 significance levels using STATA for Windows ver. 11.2 (Stata Corp., College Station, TX, USA).

Table 1. Demographic characteristics of the study population

\begin{tabular}{|c|c|c|}
\hline Characteristic & №. (\%) & Ratio of death \\
\hline Total & $6,818(100.00)$ & 8.00 \\
\hline \multicolumn{3}{|l|}{ Age group (y) } \\
\hline$<20$ & $156(2.30)$ & 5.76 \\
\hline 20-29 & $3,782(55.50)$ & 6.05 \\
\hline 30-39 & $2,185(32.00)$ & 8.51 \\
\hline $40-49$ & $415(6.10)$ & 11.80 \\
\hline $50-59$ & $178(2.10)$ & 31.20 \\
\hline$\geq 60$ & $102(1.50)$ & 43.75 \\
\hline \multicolumn{3}{|l|}{ Gender } \\
\hline Female & $3,661(53.70)$ & 7.94 \\
\hline Male & 3,157 (46.30) & 8.07 \\
\hline \multicolumn{3}{|l|}{ Occupation } \\
\hline Homemakers & 2,220 (32.60) & 8.55 \\
\hline Unemployed & $2,143(31.40)$ & 8.67 \\
\hline Student & $1,188(17.40)$ & 3.50 \\
\hline Employed $^{*}$ & 910 (13.30) & 9.01 \\
\hline Other ${ }^{\dagger}$ & $357(5.30)$ & 5.88 \\
\hline \multicolumn{3}{|l|}{ Educational level } \\
\hline Illiterate & $773(11.30)$ & 17.20 \\
\hline Primary school & $401(5.90)$ & 20.44 \\
\hline Guidance/high school & $1,988(29.20)$ & 7.89 \\
\hline Diploma & $2,775(40.70)$ & 4.90 \\
\hline University & $881(12.29)$ & 4.31 \\
\hline \multicolumn{3}{|l|}{ Marital status } \\
\hline Married & $2,937(43.10)$ & 9.83 \\
\hline Single & $3,881(56.90)$ & 6.62 \\
\hline \multicolumn{3}{|l|}{ Socioeconomic status ${ }^{\ddagger}$} \\
\hline Lowest & $95(1.40)$ & 27.36 \\
\hline Second-to-lowest & $295(4.30)$ & 23.70 \\
\hline Middle & $1,382(20.30)$ & 12.08 \\
\hline Second-to-highest & $4,004(58.70)$ & 6.89 \\
\hline Highest & $1,041(15.30)$ & 2.40 \\
\hline
\end{tabular}

${ }^{*}$ Employed included employees and free jobs. ${ }^{\dagger}$ Other included soldiers, retirees, workers, etc. ${ }^{\ddagger}$ Socioeconomic status based on a composite measure of household income and educational achievement.

\section{RESULTS}

The total number of suicide attempts during the five-year study period was 6,818 , and the ratio of deaths to attempters was 0.08 (95\% confidence interval [CI], 0.073 to 0.086 ). The greatest proportion of cases were in the 20-29-year-old age group (55.5\%) and those aged 60 years and older showed the lowest proportion (i.e., 1.5\%). A total of 6,818 cases $(53.7 \%)$ were female and slightly more than half (56.9\%) were single. Subjects who finished high school and those with a diploma were more likely to attempt suicide (40.7\%). Homemakers accounted for more than three quarters of the subjects investigated. Most suicide attempters reported a moderate to high SES (94.3\%) and only 5.7\% were found to have lower SES (Table 1).

The correlates of study population characteristics and suicidal behaviors are presented in Table 2. Logistic regression was used to examine the risk of sociodemographic status in prior suicide attempts and

Table 2. Sociodemographic correlates of suicide behaviors (prior suicide attempts and suicide deaths)

\begin{tabular}{|c|c|c|c|c|}
\hline Variable & $\begin{array}{l}\text { Prior suicide } \\
\text { attempt }\end{array}$ & P-value & Suicide death & P-value \\
\hline \multicolumn{5}{|l|}{ Age group (y) } \\
\hline$<20$ & 1.00 & & 1.00 & \\
\hline $20-29$ & $1.22(0.56-2.62)$ & 0.078 & $1.22(0.48-2.15)$ & 0.068 \\
\hline $30-39$ & $1.06(0.45-2.46)$ & 0.060 & $2.22(1.30-4.77)$ & 0.001 \\
\hline $40-49$ & $1.42(0.88-3.23)$ & 0.063 & $3.09(1.96-6.93)$ & 0.001 \\
\hline $50-59$ & $1.08(0.92-4.06)$ & 0.052 & $6.14(2.77-9.63)$ & 0.001 \\
\hline$\geq 60$ & $1.88(1,03-7.12)$ & 0.036 & $6.33(3,85-11.91)$ & 0.001 \\
\hline \multicolumn{5}{|l|}{ Gender } \\
\hline Female & 1.00 & & 1.00 & \\
\hline Male & $1.31(1.03-2.18)$ & 0.001 & $3.66(3.03-4.11)$ & 0.001 \\
\hline \multicolumn{5}{|l|}{ Occupation } \\
\hline Housewife & 1.00 & & 1.00 & \\
\hline Unemployed & $1.31(0.72-1.46)$ & 0.091 & $1.58(1.12-2.30)$ & 0.001 \\
\hline Student & $2.02(1.05-3.88)$ & 0.033 & 1.70 (1.07-2.73) & 0.075 \\
\hline Working* & $2.93(0.90-9.46)$ & 0.125 & $0.88(0.43-1.23)$ & 0.093 \\
\hline Other ${ }^{\dagger}$ & $2.93(0.87-6.75)$ & 0.087 & $1.26(0.95-1.56)$ & 0.071 \\
\hline \multicolumn{5}{|l|}{ Educational level } \\
\hline Illiterate & 1.00 & & 1.00 & \\
\hline Primary school & $0.93(0.52-1.67)$ & 0.128 & $2.03(1.42-4.07)$ & 0.001 \\
\hline Guidance/high school & $0.70(0.44-1.11)$ & 0.820 & $1.88(0.44-2.88)$ & 0.001 \\
\hline Diploma & $2.13(1.18-2.80)$ & 0.001 & $3.40(2.39-5.06)$ & 0.001 \\
\hline University & $0.98(0.77-3.77)$ & 0.123 & 2.98 (1.77-4.39) & 0.001 \\
\hline \multicolumn{5}{|l|}{ Marital status } \\
\hline Married & 1.00 & & 1.00 & \\
\hline Single & $1.62(1.22-4.11)$ & 0.001 & $2.38(1.87-3.95)$ & 0.001 \\
\hline \multicolumn{5}{|c|}{ Socioeconomic status ${ }^{\ddagger}$} \\
\hline Lowest & 1.00 & & 1.00 & \\
\hline Second-to-lowest & $0.28(0.13-2.35)$ & 0.440 & $0.71(0.26-1.91)$ & 0.502 \\
\hline Middle & $0.22(0.63-2.36)$ & 0.550 & $1.15(0.36-9.06)$ & 0.810 \\
\hline Second-to-highest & $0.73(0.17-2.55)$ & 0.222 & $3.32(0.42-7.36)$ & 0.495 \\
\hline Highest & $0.05(0.05-2.14)$ & 0.234 & $3.55(0.80-15.68)$ & 0.048 \\
\hline
\end{tabular}

Values are presented as odds ratio (95\% confidence interval). Derived from multiple logistic regression models adjusted for demographic variables, age, gender, marital status, education, employment, and socioeconomic status.

${ }^{*}$ Employed included employee and free jobs. ${ }^{\dagger}$ Other included soldiers, retirees, workers, etc. ₹Socioeconomic status based on principal component analysis. 
suicide deaths. According to the result, the odds ratio (OR) of prior suicide attempts and suicide deaths was higher among single individuals with prior suicide attempts compared to married people (OR, 1.62; 95\% CI, 1.22 to 2.06; $\mathrm{P}=0.001$ ) and ( $\mathrm{OR}, 2.38 ; 95 \% \mathrm{CI}, 1.77$ to 4.39 ; $\mathrm{P}=0.001)$, respectively. The adjusted $\mathrm{OR}$ estimates of prior suicide attempts and suicide deaths in males versus females were (OR, 1.31; 95\% CI, 1.03 to 2.28; $\mathrm{P}=0.001$ ) and (OR, 3.66; 95\% CI, 3.03 to 4.11; $\mathrm{P}=0.001$ ), respectively. Significant excess risk of prior suicide attempts also appeared for those with a diploma versus illiterate persons (OR, 2.13; $95 \% \mathrm{CI}, 1.18$ to 2.80 ; $\mathrm{P}=0.001$ ). Other occupations were not significantly related to a higher risk of prior suicide attempts compared with housewives.

As shown in Table 2, the OR of prior suicide attempts and suicide deaths was higher among older age groups than younger age groups. We also found that prior suicide attempts and suicide deaths were significantly associated with SES. Those with higher SES (second-tohighest level and above) were significantly less likely to report any form of suicidal behaviors than those with the lowest SES.

The slope index of the ACI is shown in Figure 1. This figure displays suicide attempters with lower SES having more risk of death from sui-

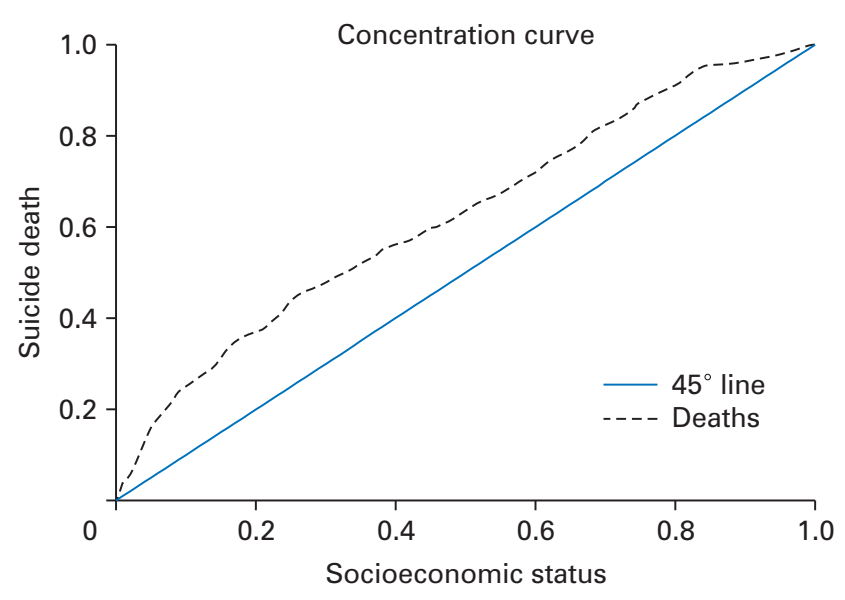

Figure 1. Absolute concentration curve of suicide deaths.

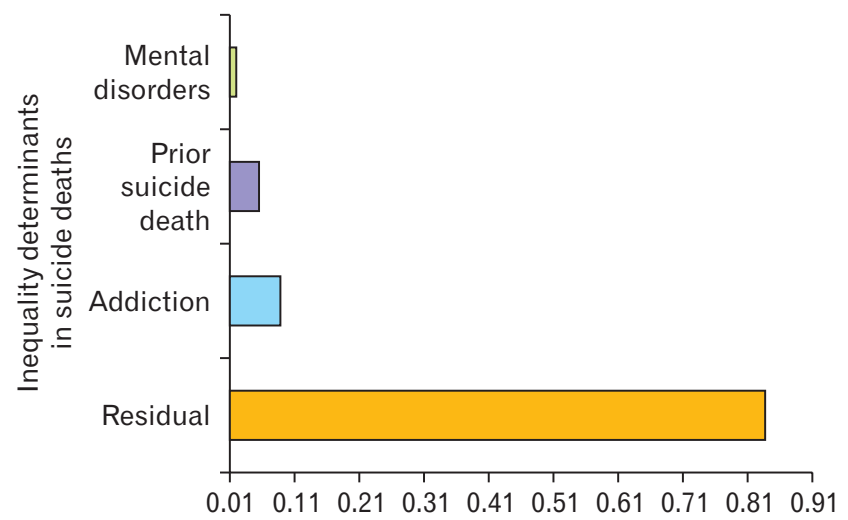

Figure 2. Contributions of socioeconomic determinants in inequality of suicide deaths. cide. Disparities existed in deaths across socioeconomic groups. The overall ACI for suicide deaths was -0.352 (95\% CI, -0.389 to 0.301 ). The height of the bar corresponded to the severity of socioeconomic inequality in suicide deaths.

Through the decomposition method, we created a dummy variable for all variables with several categories (Figure 2). This figure shows the relative contribution indices of the explanatory variables to allow the computation of the 'contributions' of each of the variables. According to the results, $9.8 \%$ of the socioeconomic inequality in suicide deaths was due to addiction, $0.04 \%$ of the inequality was due to mental disorders, $6.4 \%$ was the result of prior suicide deaths, and $83 \%$ was the residual component, which means it could not be explained by the data entered into the model.

The overall socioeconomic inequality in suicide deaths across the province through 2010-2014 is shown in Figure 3. The ACI ranged from -0.34 to -0.03 and was significantly lower than 1 for all years of the study period, implying higher suicide deaths among people with the lowest SES compared with the highest. According to the time trend analysis, inequality in suicide deaths have been declined over time of the study.

\section{DISCUSSION}

Consistent with previous reports, ${ }^{20,21)}$ the current study found that women had a greater likelihood of attempting suicide ( $49.4 \%$ versus $42.6 \%)$, whereas men had a higher rate of suicide deaths (8.1\% versus $7.9 \%$ ). One potential explanation may be the difference in methods of attempting suicide among males and females. For example, the most common methods of attempting suicide used by males in Iran involve hanging and firearms, which have higher fatality rates compared with the self-burning method commonly used by females. This finding differs from those reported in China and India. ${ }^{21,22)}$ Substantial research suggests prior suicide attempts are the greatest predictor of future suicide attempts or suicide deaths. ${ }^{4,20)}$ A meta-analysis of psychological autopsy studies found suicide attempts and deliberate self-harm to be the most significant risk factors associated with suicidal behavior. ${ }^{23)}$

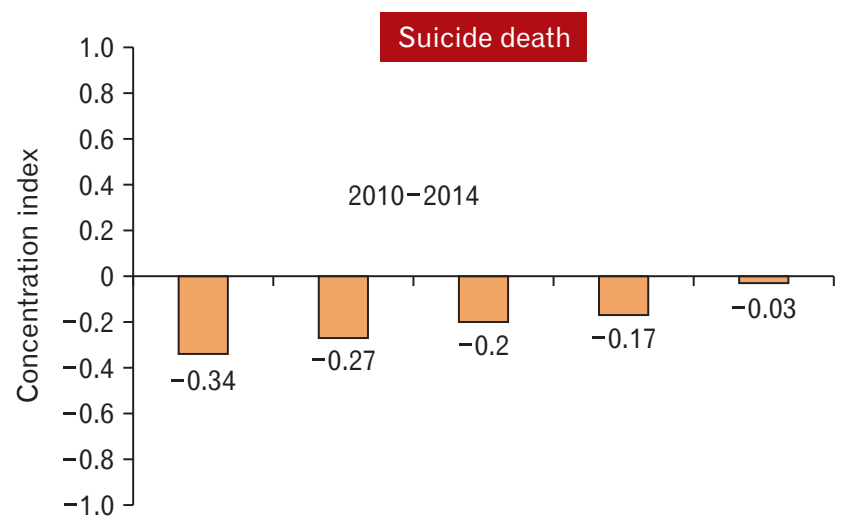

Figure 3. Trends in the concentration indices of suicide deaths in llam Province (2010-2014). 
Similarly, findings from this study suggest a prior suicide attempt to be a prominent risk factor for suicide, and excess risk among males and attempters with better educational levels (diploma compared with illiterate).

In this study, we used SRSD to determine inequality in suicide deaths in attempters. The findings from the current study showed that death is not equal in all SES groups and suicide deaths are more common in lower SES groups. The results of this study are in line with previous ecological studies investigating the relationship between socioeconomic characteristics and suicide rates, in particular those studies focused on high-income settings. ${ }^{24-26)}$ A systematic review by Rehkopf and Buka ${ }^{27)}$ found that, among studies with statistically significant results, $50 \%$ and $73 \%$ of studies reported an inverse relation between income and educational characteristics and suicide deaths, respectively. They also found that the probability of reporting an inverse relationship between social rank and suicide mortality was higher among studies conducted in Asia (94\% of studies with statistically significant results). Similar findings were reported by another study focusing on countries in the Eastern Mediterranean region (where Iran is located), indicating that high-income countries had lower suicide mortality rates than their low-income and middle-income counterparts. ${ }^{28)}$ It is argued that people in provinces with lower social rank generally have more adverse experiences, poorer mental health, lower access to psychiatric services, and lower access to health facilities. Therefore, our findings are consistent with these reports that have shown suicide deaths to be more common in community with lower socioeconomic groups. This result suggests that concentrating prevention programs on these people can potentially reduce inequality and suicide deaths.

In addition, we found both absolute and relative inequalities in suicide deaths according to SES were reduced over time. Thus, while the prevalence of suicide attempts and suicide deaths increased over time, the rates of inequality in suicide deaths declined. One potential explanation is that actual inequality was not reduced over time in Ilam Province, but that the rate of death from suicide increased in better socioeconomic groups in recent years. Another plausible explanation is that suicide attempts in better socioeconomic areas became more prevalent, reflecting a silent epidemic.

Policy makers can use the decomposition method for planning and policy purposes. In this study, addiction was responsible for the highest positive contribution to inequality. However, addiction is more prevalent in lower socioeconomic groups; thus, policy makers should pursue programs to enhance the social welfare of this group, to decrease the positive contribution of addiction in the socioeconomic inequality of suicide deaths.

An important limitation of our study is that due to its cross-sectional nature, inference regarding the significant relationship between SES and suicide deaths is not conclusive. Second, we were limited to straight data, such as income, to measure socioeconomic scores.

In summary, we used the inequality approach to expand study of the connection between SES and suicide death. The present study's findings showed suicide deaths were distributed unequally, confirm- ing the gap between advantaged and disadvantaged attempters. Socioeconomic inequalities in suicide deaths tended to diminish over time, while suicide attempts progressed in Ilam Province.

\section{CONFLICT OF INTEREST}

No potential conflict of interest relevant to this article was reported.

\section{ACKNOWLEDGMENTS}

We would like to thank Vice-Chancellor of Research and Technology of Ilam University of Medical Sciences for financial support of this study.

\section{REFERENCES}

1. Kessler RC, Berglund P, Borges G, Nock M, Wang PS. Trends in suicide ideation, plans, gestures, and attempts in the United States, 1990-1992 to 2001-2003. JAMA 2005;293:2487-95.

2. Malakouti SK, Davoudi F, Khalid S, Ahmadzad Asl M, Moosa Khan M, Alirezaei N, et al. The epidemiology of suicide behaviors among the countries of the Eastern Mediterranean Region of WHO: a systematic review. Acta Med Iran 2015;53:257-65.

3. Nock MK, Borges G, Bromet EJ, Cha CB, Kessler RC, Lee S. Suicide and suicidal behavior. Epidemiol Rev 2008;30:133-54.

4. Harris EC, Barraclough B. Suicide as an outcome for mental disorders: a meta-analysis. Br J Psychiatry 1997;170:205-28.

5. Tiihonen J, Lonnqvist J, Wahlbeck K, Klaukka T, Tanskanen A, Haukka J. Antidepressants and the risk of suicide, attempted suicide, and overall mortality in a nationwide cohort. Arch Gen Psychiatry 2006;63: 1358-67.

6. Beautrais AL, Joyce PR, Mulder RT, Fergusson DM, Deavoll BJ, Nightingale SK. Prevalence and comorbidity of mental disorders in persons making serious suicide attempts: a case-control study. Am J Psychiatry 1996;153:1009-14.

7. Veisani Y, Delpisheh A. Decomposing of socioeconomic inequality in mental health: a cross-sectional study into female-headed households. J Res Health Sci 2015;15:218-22.

8. Delpisheh A, Topping J, Reyad M, Tang AW, Brabin BJ. Smoking exposure in pregnancy: use of salivary cotinine in monitoring. Br J Midwifery 2007;15:216-20.

9. Veisani Y, Delpisheh A, Sayehmiri K, Moradi G, Hassanzadeh J. Associations of suicide seasonality with rural-urban residence and mental disorders in Ilam, Iran. Iran J Med Sci 2016;41:461-2.

10. Burrows S, Laflamme L. Socioeconomic disparities and attempted suicide: state of knowledge and implications for research and prevention. Int J Inj Contr Saf Promot 2010;17:23-40.

11. Yoder KA, Hoyt DR. Family economic pressure and adolescent suicidal ideation: application of the family stress model. Suicide Life Threat Behav 2005;35:251-64.

12. Chan WS, Law CK, Liu KY, Wong PW, Law YW, Yip PS. Suicidality in Chinese adolescents in Hong Kong: the role of family and cultural influences. Soc Psychiatry Psychiatr Epidemiol 2009;44:278-84.

13. Maki NE, Martikainen PT. Socioeconomic differences in suicide mor- 
tality by sex in Finland in 1971-2000: a register-based study of trends, levels, and life expectancy differences. Scand J Public Health 2007;35: 387-95.

14. Veisani Y, Delpisheh A, Sayehmiri K, Moradi G, Hassanzadeh J. Suicide attempts in Ilam Province, Western Iran, 2010-2014: a time trend study. J Res Health Sci 2016;16:64-7.

15. Vyas S, Kumaranayake L. Constructing socio-economic status indices: how to use principal components analysis. Health Policy Plan 2006;21: 459-68.

16. Wagstaff A, Paci P, van Doorslaer E. On the measurement of inequalities in health. Soc Sci Med 1991;33:545-57.

17. Wagstaff A, van Doorslaer E, Paci P. Equity in the finance and delivery of health care: some tentative cross-country comparisons. Oxf Rev Econ Policy 1989;5:89-112.

18. Wagstaff A, van Doorslaer E, Watanabe N. On decomposing the causes of health sector inequalities with an application to malnutrition inequalities in Vietnam. J Econom 2003;112:207-23.

19. Speybroeck N, Konings P, Lynch J, Harper S, Berkvens D, Lorant V, et al. Decomposing socioeconomic health inequalities. Int J Public Health 2010;55:347-51.

20. Oquendo MA, Bongiovi-Garcia ME, Galfalvy H, Goldberg PH, Grunebaum MF, Burke AK, et al. Sex differences in clinical predictors of suicidal acts after major depression: a prospective study. Am J Psychiatry 2007;164:134-41.
21. Patel V, Ramasundarahettige C, Vijayakumar L, Thakur JS, Gajalakshmi V, Gururaj G, et al. Suicide mortality in India: a nationally representative survey. Lancet 2012;379:2343-51.

22. Phillips MR, Cheng HG. The changing global face of suicide. Lancet 2012;379:2318-9.

23. Yoshimasu K, Kiyohara C, Miyashita K; Stress Research Group of the Japanese Society for Hygiene. Suicidal risk factors and completed suicide: meta-analyses based on psychological autopsy studies. Environ Health Prev Med 2008;13:243-56.

24. Clouston SA, Rubin MS, Colen CG, Link BG. Social inequalities in suicide: the role of selective serotonin reuptake inhibitors. Am J Epidemiol 2014;180:696-704.

25. Pompili M, Innamorati M, Vichi M, Masocco M, Vanacore N, Lester D, et al. Inequalities and impact of socioeconomic-cultural factors in suicide rates across Italy. Crisis 2011;32:178-85.

26. Kiadaliri AA, Saadat S, Shahnavazi H, Haghparast-Bidgoli H. Overall, gender and social inequalities in suicide mortality in Iran, 2006-2010: a time trend province-level study. BMJ Open 2014;4:e005227.

27. Rehkopf DH, Buka SL. The association between suicide and the socioeconomic characteristics of geographical areas: a systematic review. Psychol Med 2006;36:145-57.

28. Rezaeian M. Age and sex suicide rates in the Eastern Mediterranean Region based on global burden of disease estimates for 2000. East Mediterr Health J 2007;13:953-60. 